\title{
Prominence of Different Routing Protocols in MANET for Heterogeneous Communication Environment
}

\author{
Abu Bony Amin \\ Department of Electrical \& Electronic Engineering \\ Chittagong University of Engineering and \\ Technology (CUET), Chittagong-4349, \\ Bangladesh
}

\author{
Choton Kanti Das \\ Department of Electrical \& Electronic Engineering \\ Chittagong University of Engineering and \\ Technology (CUET), Chittagong-4349, \\ Bangladesh
}

\begin{abstract}
Traffic is increasing in all types of communication day by day. The challenge of maintaining seamless communication by avoiding congestion in a cost effective way has garnered much interest of present researchers. This challenge can be met by developing solutions for communication network based on internet technologies. This is done by developing Mobile Ad hoc Network (MANET). Mobile Ad hoc networks (MANETs) are infrastructure-less dynamic networks allowing mobile nodes to communicate beyond transmission range where mobile nodes act as gateway itself. In this research new network architecture is proposed which is combination of aeronautical and nautical communication network. This research will present a new paradigm for both aeronautical and nautical communication in air to ground and air to sea at future with mobile gateway. In this paper the performance of four routing protocols i.e. AODV, DSDV, DSR and AOMDV are compared to using various performance metrics. On the basis of these comparisons the best routing protocol for MANETs in heterogeneous communication environment has been proposed by performing simulation in Network Simulator (NS-2.35). By this approach it's possible to obtain seamless communication over the whole world.
\end{abstract}

\section{Keywords}

Ad-hoc On-demand Distance Vector (AODV), Destination Sequenced Distance Vector (DSDV), Dynamic Source Routing (DSR), Ad-hoc On-demand Multipath Distance Vector (AOMDV), Mobile Ad hoc Network (MANET), Network Simulator.

\section{INTRODUCTION}

The essentiality of mobile communications and wireless internet access is rapidly increasing for people's everyday life in this era of information technology. The growing interest by commercial, rescuer, fighter aircrafts and ships in providing internet access and cellular connectivity in the cabin has led to the emergence. Apparently, a satellite communications link is the most feasible and flexible way to keep aircrafts and ships connected to the ground throughout the journey. But with a satellite, there is always a one-way end-to-end propagation delay required for the signal to travel up and down from the satellite. This is not cost effective for bandwidth usage. So, other effective way can be applied to obtain seamless communication such like as Mobile Ad hoc network [1].

Ships at sea have to maintain a regular communication with each other or to base stations on shores. Data communications at sea nowadays depend on mainly satellite and radio media which are, however, not adequate to exchange massive information due to its own high cost and low bandwidth Ships at sea in the near future would need broad and stable maritime data communication systems like on land. So, by combining the aeronautical and nautical communication network a heterogeneous communication environment will be created in where every aircrafts and ships act as a single node and they can communicate with each other which are done by Mobile Ad hoc Network (MANET).

A Mobile Ad hoc Network (MANET) is a collection of mobile wireless nodes that can dynamically form a network to exchange information without using any pre-existing fixed network infrastructure. Additionally, MANET is a selforganized and self-configurable network where the mobile nodes move arbitrarily. The mobile nodes can receive and forward packets as a router. Each node operates not only as an end system, but also as a router to forward packets. The nodes are free to move about and organize themselves into a network. These nodes change position frequently. A pair of mobile nodes may communicate with each other either directly or indirectly with the help of the intermediate nodes in the Mobile Ad Hoc Network [2][3][4]. The ability of selfconfiguration of the nodes makes them more suitable for urgently required network connection, for example in disaster hit areas where there is no communication infrastructure. MANET is the quick remedy for any disaster situation. It is a spontaneous network which is useful when dealing with wireless devices in which some of the devices are part of this network only for the duration of a communication session [5]. The Mobile Ad Hoc Network can be used for some deliberate purposes such as to increase the transmission range, to maintain seamless communication in disaster area, to establish communication among different types of vehicle [6].

Most of the previous researches on MANET with different routing protocols have mainly investigated the horizontal and vertical scenario in a communication environment [7]. In some researches, MANET was proposed only for aeronautical environment [1]. But in this approach the MANET will be implemented in heterogeneous communication environment i.e. the combination of the aeronautical and nautical communication network. Here both the aircraft and ship in sea will act as gateway. So, in this research gateway is configured as mobile by which this approach can be applied. It is our a very little attempt to obtain a cost effective way to communicate at anytime, anywhere without using most costly Satellite link. For this purpose four routing protocols i.e. AODV, DSDV, DSR and AOMDV are used to simulate the environment in Network Simulator (NS-2.35). 


\section{GIST OF SIMULATION SCENARIO}

Since, in the MANET both the aircrafts and ships themselves act as a gateway therefore, the simulation is done for mobile gateway. Here by this network air-ground (A/G coms) as well as air-sea (A/S coms) communication can be made very effectively. The usage of Satellite link will be reduced. So, the end-to-end delay will be reduced appreciably. On the other hand a cost effective way for communication will be achieved.

Fig 1 shows that MANET is envisioned as a means to extend the coverage of air-ground access networks. By enabling aircraft and ships themselves to act as network routers, a mobile ad hoc network is formed in all over the sky and ocean. According to this scenario, every ship in the sea and every aircraft in the sky can communicate with each other without using satellite link. Here, ship to ship and ship to shore data communications also will be maintained.

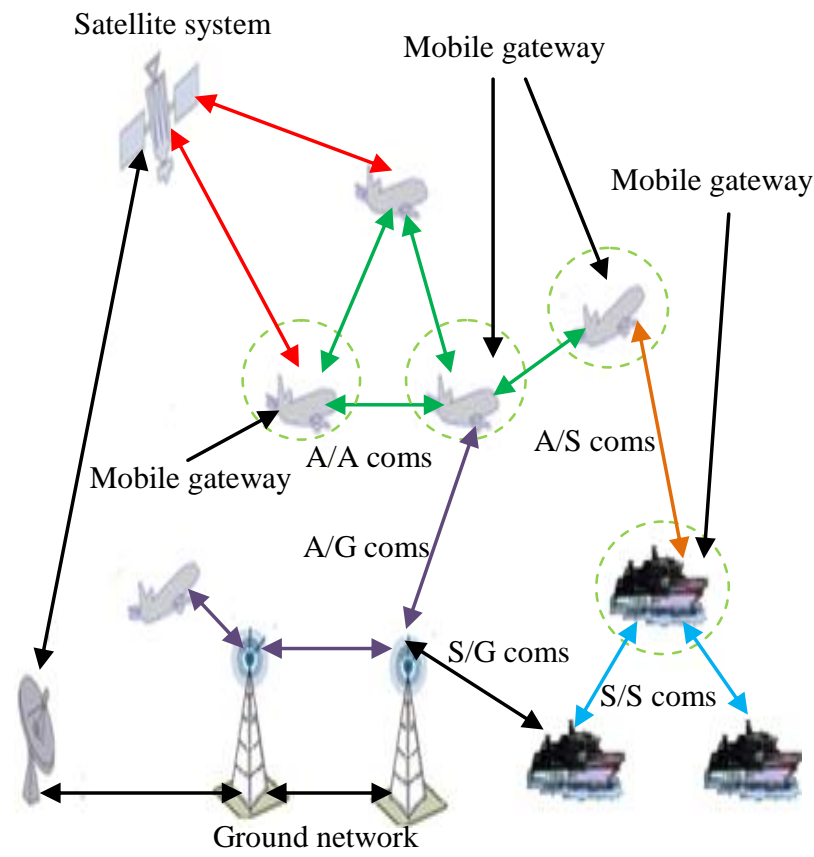

Fig 1: Scenario of air-ground (A/G coms), air-air (A/A coms), air-sea (A/S coms) and sea-sea (S/S coms) communication by Mobile Ad hoc Network with mobile gateways [8]

\section{OVERVIEW OF AODV, DSDV, DSR AND AOMDV ROUTING PROTOCOLS}

Every routing protocol has its own merits and demerits, none of them are absolutely perfect. In this paper, four routing protocols i.e. AODV, DSDV, DSR and AOMDV are taken to evaluate their performance in MANET under heterogeneous communication environment.

\subsection{Ad-hoc On-Demand Distance Vector Routing (AODV)}

AODV is a reactive protocol which embraces a very different mechanism to maintain routing information. It manipulates traditional routing tables, one entry per destination. AODV uses sequence numbers maintained at each destination to determine freshness of routing information and to prevent routing loops. All routing packets carry these sequence numbers. A noteworthy feature of AODV is the maintenance of timer-based states in each node, regarding utilization of individual routing table entries. A routing table entry would be expired if it were not used recently. A set of precursory nodes is maintained for each routing table entry, indicating the set of neighboring nodes which use that entry to route data packets. These nodes are notified with RREP (Route Reply) packets when the next-hop link breaks [2]. The AODV offers advantages by maintaining information of only active routes. So, less memory space is required. Consequently, the performance is increased. On the other hand, the disadvantage is that this protocol is not scalable and in large networks it does not perform well and does not support asymmetric links [9].

\subsection{Destination Sequenced Distance Vector (DSDV)}

Destination Sequenced Distance Vector is a loop free routing protocol whose table driven algorithm adopts the BellmanFord routing mechanism. Every routing table wields information about every potential destination from a node to any other nodes in the network and also the number of hops to each destination. By which data packets are transmitted between the nodes. The protocol has three principal properties: to avert loops, to resolve the "count to infinity" problem and to scale down high routing overhead. Each node issues a sequence number which is attached to every new routing-table update message. It uses two different types of routing-table updates, named "full" and "incremental dumps", respectively for minimizing the number of control messages broadcasted in the network. Furthermore, each node keeps statistical data pertaining the average setting time of a message that the node receives from any neighboring node. DSDV takes into account only bidirectional links between nodes. In all table driven protocols each node sustains a table that contains the next hop to reach all destinations. They are exchanged among neighboring nodes at regular intervals for keeping the tables up to date. It also occurs when substantial topology alterations are observed. The DSDV offers benefits by delaying the broadcast of a routing update by the length of the settling time [2] [9] [10].

\subsection{Dynamic Source Routing (DSR)}

The most prominent feature of DSR is the usage of source routing. Source routing is defined as that the sender knows the complete hop-by-hop routes to the destination which are stored in a route cache. The data packets carry the source route in the packet header. If a node in the ad hoc network attempts to send a data packet to a destination without knowing the route, it uses a route discovery process to dynamically ascertain such a route. Route discovery is done by flooding the network with route request (RREQ) packets and every node receiving an RREQ rebroadcasts it unless it is the destination or it has a route to the destination in its route cache. The route request (RREQ) packets contain the information about destination node including address and unique identification number of destination node. Such a node replies to the RREQ with a route reply (RREP) packet that is routed back to the original source. RREQ and RREP packets are also source routed. The RREQ builds up the path traversed across the network and the RREP routes itself back to the source by traversing this path backward. The route carried back by the RREP packet is cached at the source for future use. If any link on a source route is broken, the source node is notified using a route error (RERR) packet. The source 
removes any route using this link from its cache. A new route discovery process must be originated by the source if this route is still needed. DSR makes very aggressive utilization of source routing and route caching [2] [9].

\subsection{Ad-hoc On-demand Multipath Distance Vector Routing (AOMDV)}

Ad-hoc On-demand Multipath Distance Vector Routing (AOMDV) protocol is an extension to the AODV protocol which computes multiple loop-free and link disjoint paths. The routing entries for each destination contain a list of the next-hops along with the corresponding hop counts. Eventually, all the next hops have the same sequence number. This helps in keeping track of a route. For each destination, a node maintains the advertised hop count, which is defined as the maximum hop count for all the paths. This hop count is used for sending route advertisements of the destination. Each duplicate route advertisement received by a node defines a substitute path to the destination. Loop freedom is ensured for a node by accepting substitute paths to destination when it has a less hop count than the advertised hop count for that destination. Since, the maximum hop count is used the advertised hop count does not alter for the same sequence number the next hop list and the advertised hop count are reinitialized after receiving a route advertisement for a destination with a greater sequence number. AOMDV can be utilized to detect node-disjoint or link-disjoint routes. To find node-disjoint routes, each node does not immediately refuse duplicate RREQs. Nodes cannot be broadcast duplicate RREQs, so any two RREQs arriving at an intermediate node via a different neighbor of the source could not have traversed the same node. Hence, Each RREQs arriving via a different neighbor of the source defines a node-disjoint path. The advantage of using AOMDV is that it allows intermediate nodes to reply to RREQs, while still selecting disjoint paths. The AOMDV has a great privilege. Because, it allows intermediate nodes to reply to RREQs, while still selecting disjoint paths. However, AOMDV has more message overheads during route discovery due to increased flooding and since it is a multipath routing protocol, the destination replies to the multiple RREQs those results are in longer overhead [9].

\section{SIMULATION SETUP}

The network performances such as throughput, packets dropped, packet delivery ratio, end-to-end delay, packets received and packets forwarded were evaluated using NS-2 simulator. The entire simulations were accomplished by using NS-2.35 network simulator which is a discrete event driven simulator [11]. This section describes the network scenario, the movement model, the communication model, and the simulation parameters that used in the network topology.

\subsection{Network Scenario}

The simulated network is spanning in a standard area of $1000 \times 1000 \mathrm{~m}^{2}$. Each mobile node in our simulation has a wireless transmission range of 250 meter, which is the standard range and also used by the other research works [12]. In this simulation scenario 2 gateways are considered in the MANET in order to load balance the Internet traffic. Both of the gateways are configured as mobile gateway. A higher Internet bandwidth for gateways compared to that of the MANET nodes is assumed. Simulations are run for 200 units of simulation time. A screenshot of a simulation scenario is given in Fig 2. In the figure, the green marked circular node represents the gateways, whose node id is 14 .The node 7 is also marked as gateway because, it was acting as a gateway at previous operation. Both the gateways are mobile. Node 1 and node 13 act as source and destination respectively. In this scenario, Node 4, 5, 6, 7, 14 are configured as mobile nodes.

In this network topology the number of nodes is kept constant but the mobility of nodes is varied for different routing protocols.

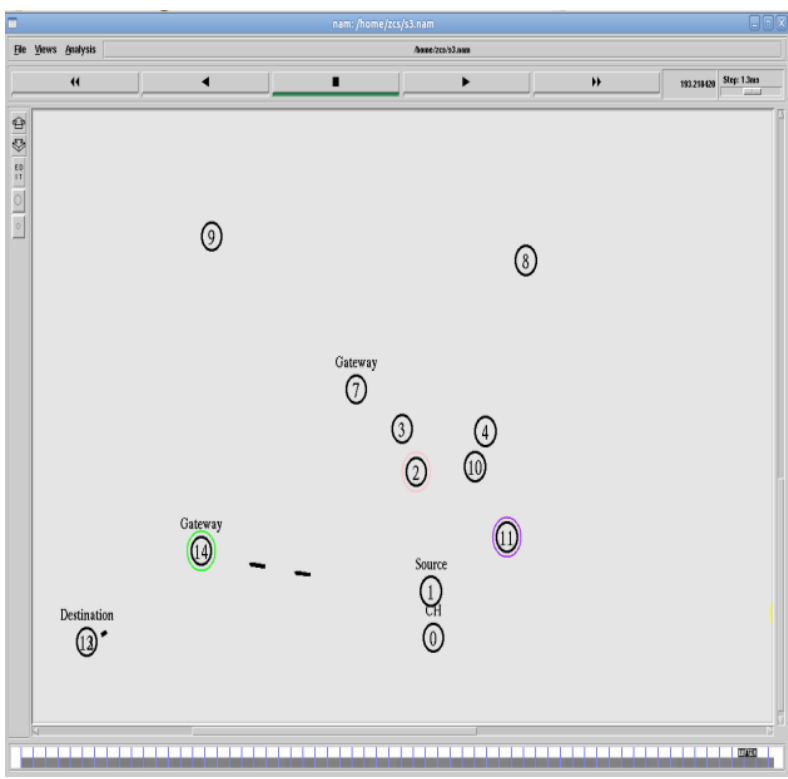

Fig 2: Screenshot of a simulation scenario in NS-2 NAM window

\subsection{Movement Model}

The random waypoint is deactivated in the Movement Model and the velocity and coordinate of node as the mobility model are manually defined for the simulation. In this model, mobile node remains stationary for a certain period called pause time. When the pause time is over, the node moves toward the defined destination at a defined speed. The different types of speeds such as $0,5,10,15,20,25 \mathrm{~m} / \mathrm{s}$ are set for different scenarios. When the node reaches the destination, it again remains stationary for the pause time period and repeats the same procedure until the end of the simulation. The pause time is set to 20 seconds in our simulations which is good enough for a node to change the movement direction.

\subsection{Communication Model}

In this simulation, each mobile node uses Transmission Control Protocol (TCP) traffic to send packets to the corresponding hosts in the Internet. Each mobile node is permitted to generate packets of size 512 bytes in the MANET. By varying the velocity of nodes, the characteristics of different routing protocol are actually observed in Mobile Ad Hoc Network with different simulation scenarios.

\subsection{Mobility Generation Models}

The movement scenario can be defined for each simulation. To study the effect of mobility, the simulation is carried out with movement patterns generated for different velocity of mobile nodes. In this simulation, the velocity of nodes is defined as $0,5,10,15,20,25 \mathrm{~m} / \mathrm{s}$ respectively. The 'setdest' command of NS-2 simulators is used to generate node- 
movement. By using 'setdest' the next destination of the mobile node and its velocity can be defined manually.

\subsection{Parameters}

Table I gives the values of simulation parameters that are used for the simulation scenarios.

Table 1. Common parameters used in the simulation scenarios $[13]$

\begin{tabular}{|c|c|}
\hline Parameter & Value \\
\hline Number of nodes & 15 \\
\hline Number of gateways & 2 \\
\hline Number of hosts & 2 \\
\hline Number of mobile nodes & 11 \\
\hline Topology size & 1000 x 1000 $\mathrm{m}^{2}$ \\
\hline Transmission range & $250 \mathrm{~m}$ \\
\hline Channel type & Wireless \\
\hline MAC type & TC2.11 \\
\hline Traffic type & 512 bytes \\
\hline Packet size & AODV/DSDV/DSR/AOMDV \\
\hline Routing protocol & $200 \mathrm{~s}$ \\
\hline Pause time & \\
\hline Simulation time & \\
\hline
\end{tabular}

\section{SIMULATION DETAILS}

When the node speed is set to $5 \mathrm{~m} / \mathrm{s}$ for these routing protocols firstly the source discover the node 7 as a gateway. But node 7 was a mobile gateway. It moved away from the source. So, the source lost the gateway. At that time, the node 7 dropped the packets which are sent from node 1. Fig 3 depicts this occurrence.

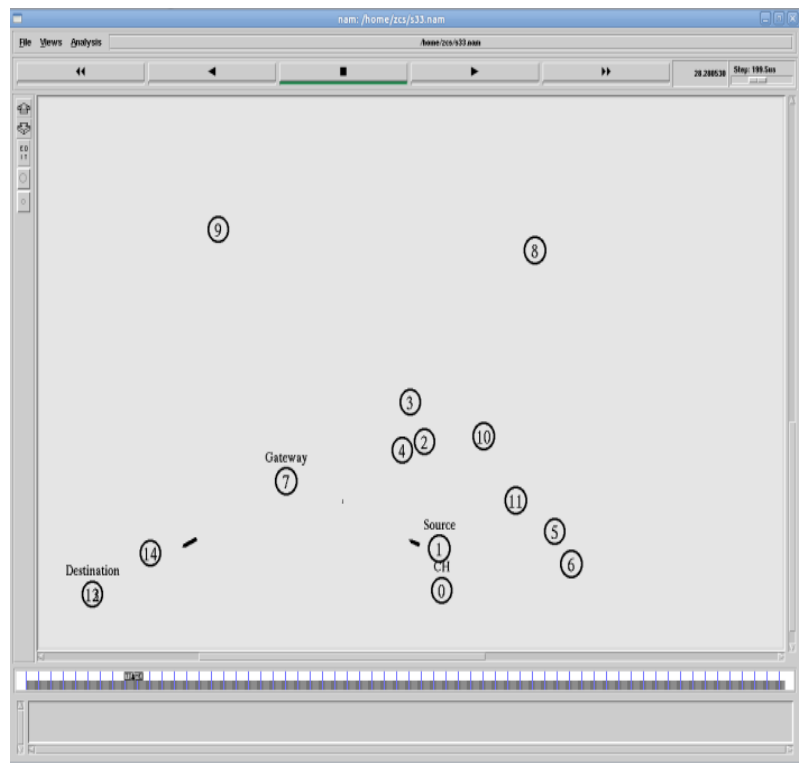

Fig 3: Screenshot of a NAM window when node 7 acts as a gateway

After losing node 7 as a gateway the node 1 is searching for new gateway for sending packet to the destination node 13.But at this time only some node will advertise itself to the node 1 which is shown in Figure 4. It is noticeable from Fig 4 that, node 8 and node 9 are not advertising themselves to act as a gateway.

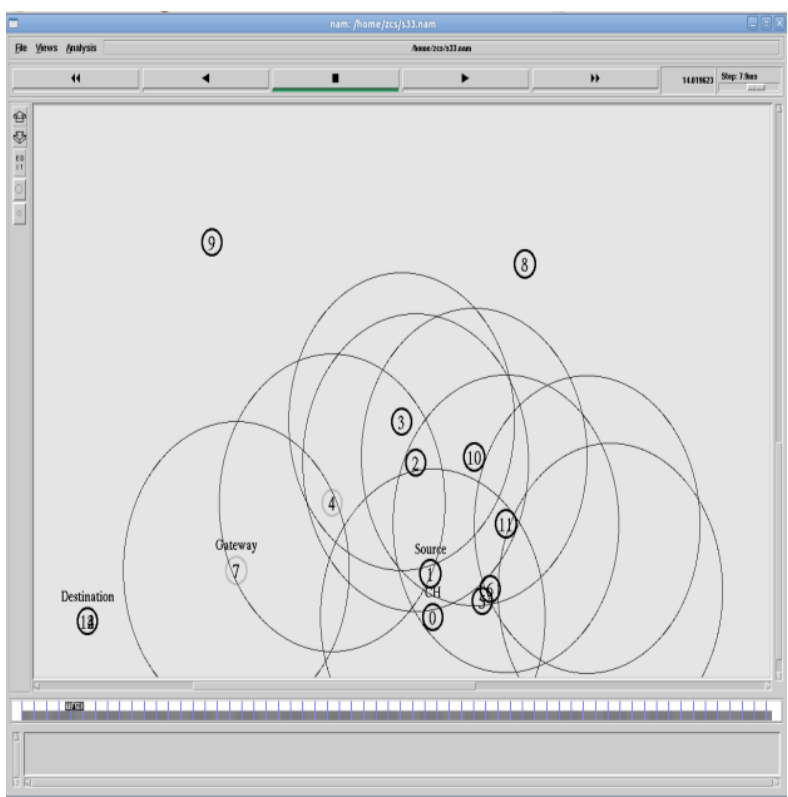

Fig 4: Screenshot of a NAM window when node 1 searching for gateway

Fig 5 depicts that when node 14 is not ready to become a gateway, the former gateway node 7 is starting to drop the packet which are sent from the source node1. So the packet losses have occurred there. But, if the velocity of node 14 is increased, then the amount of packet loss is decreases.

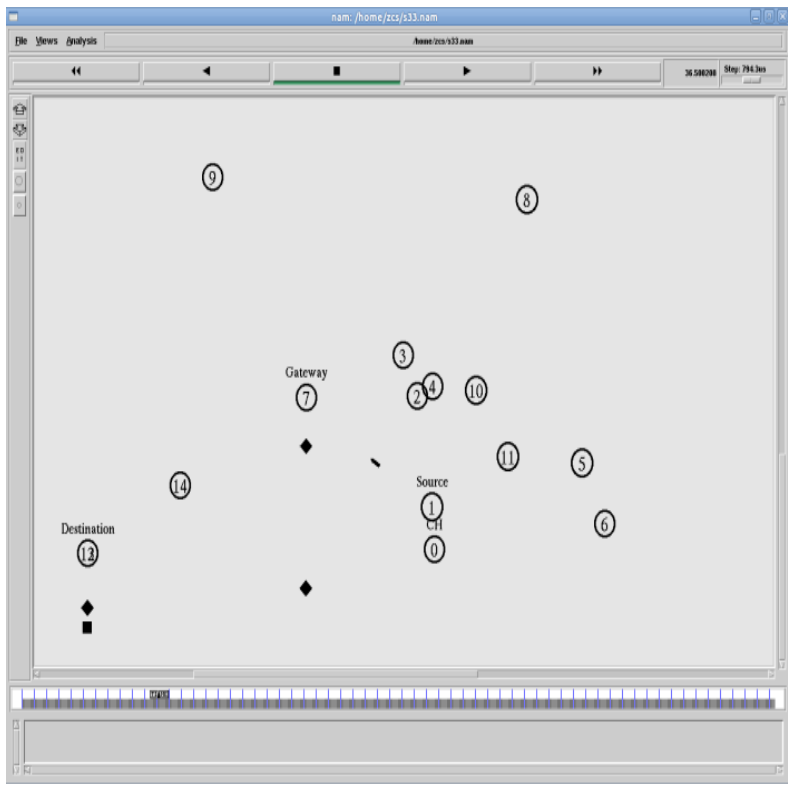

Fig 5: Screenshot of a NAM window when node 7 drops the packets 
At last, the node 14 moves toward the source node slowly. So, the source node gets the node 14 at its sight which is shown in Fig 6. In this scenario, finally the node 14 is selected as gateway. So the packet transfer is performed from source node 1 to destination node 13 via the gateway node 14 .

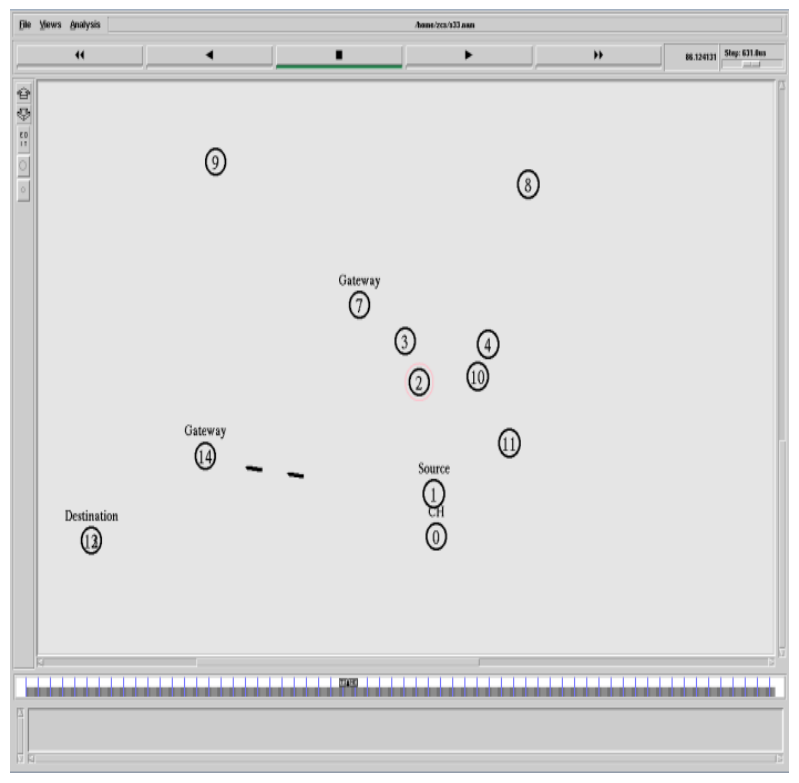

Fig 6: Screenshot of a Nam window when node 14 selected as a gateway.

\section{SIMULATION RESULT}

The simulation results are shown in the following section in the form of line graphs. Fig. 7 to Fig. 11 represents the graphs which show the comparison among different types of protocols in MANET.

\subsection{Throughput}

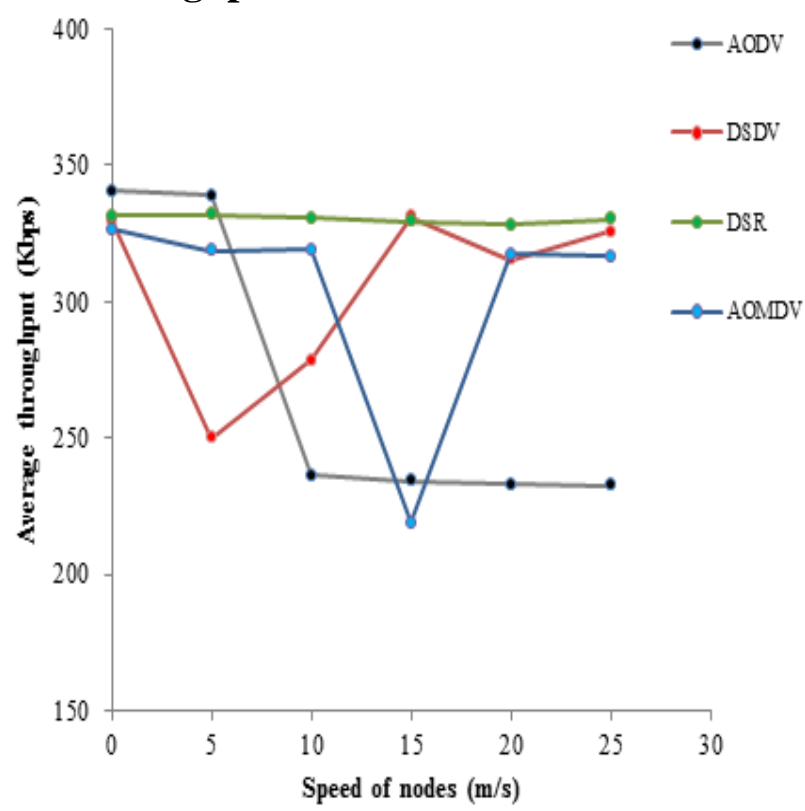

Fig 7: Average throughput vs. Speed of nodes for different routing protocol
Fig 7 depicts that, DSR shows good and more stable throughput as compare to other protocols AODV, DSDV and AOMDV. The throughput of DSR is near to same for any mobility. Throughput of DSDV is very poor at lower mobility. It is noticeable from the figure, that the throughput of the AODV and AOMDV routing protocol decreases with mobility increment more abruptly. So, for higher mobility performance of the DSR protocol is better.

\subsection{Packet Delivery Ratio(r/s)}

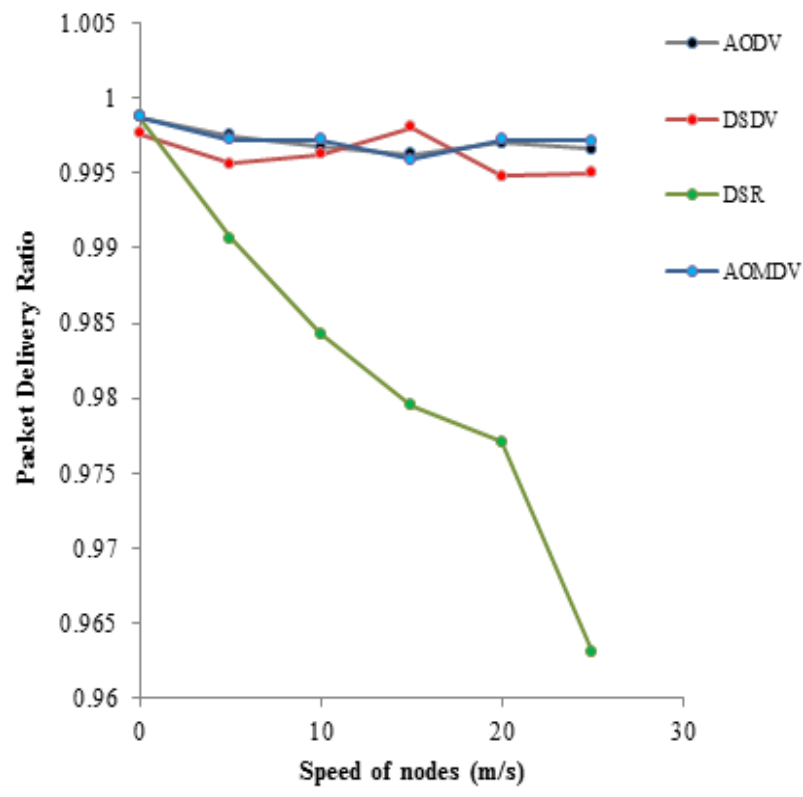

Fig 8: Packet Delivery Ratio vs. Speed of nodes for different routing protocol

It is clear from Fig 8 that, the packet delivery ratio of DSR is very less as compared to other routing protocols. The performance of DSDV is not good for very high mobility. But both the AODV and AOMDV show better performance than other two routing protocols. The reason for having better packet delivery ratio of AODV and AOMDV routing protocols is that they allow packets to stay in the send buffer for route discovery and once the route is discovered, data packets are sent on that route to be delivered at the destination.

\subsection{End-to-End Delay}




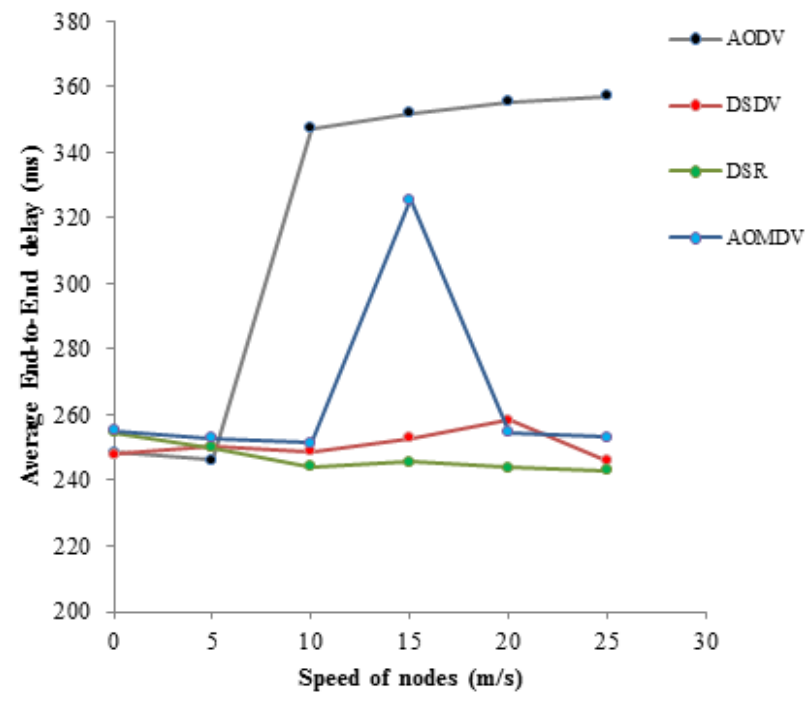

Fig 9: Average End-to-End delay vs. Speed of nodes for different routing protocol

Fig 9 depicts the performance of different types of routing protocols for End-to-End delay. On comparing the graphs it can be observed that delay in DSR routing protocol is quite low as compare to AODV, DSDV and AOMDV. The End-toEnd delay of DSDV and AODV routing protocol is better for low mobility, but not good for higher mobility. However, high end-to-end delay can be justified as AODV and DSDV deliver more packets at the destination as compared to DSR because this protocol tries to provide some sort of guarantee for the packets to be delivered at the destination by compromising at the cost of delay.

\subsection{Packets Received}

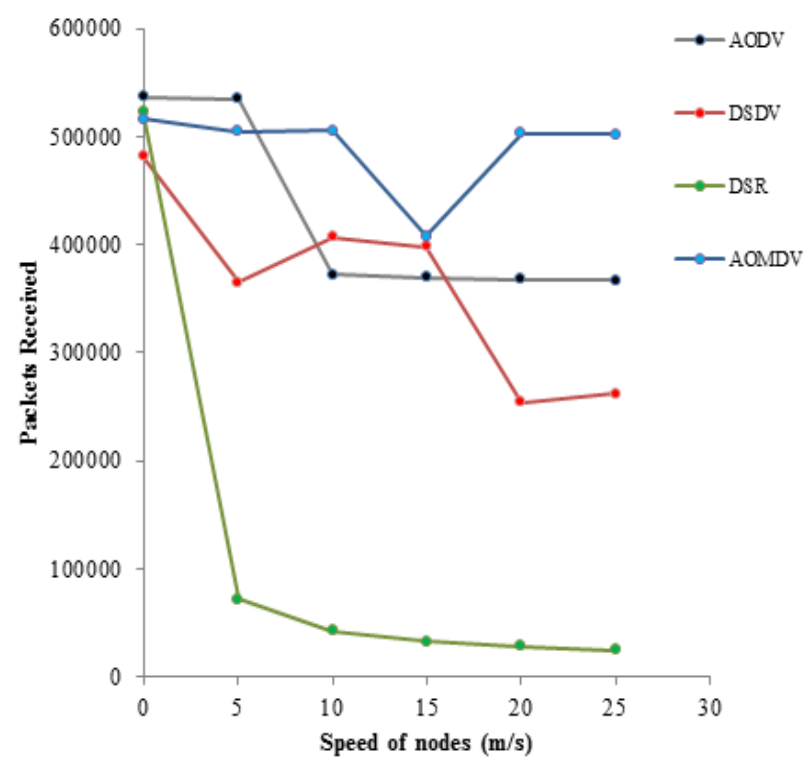

Fig 10: Packets received vs. Speed of nodes for different routing protocol

It is noticeable from Fig 10 that, the amount of packet received in AOMDV is better compared to other three routing protocols. It is shown from the graph that the amount of packet received is decreases with increasing mobility of nodes for AODV, DSDV and DSR routing protocol. Even for DSR the amount of packet received decreases abruptly with increasing mobility of nodes.

So, the performance of AOMDV routing protocol is better among all of the routing protocols for receiving packet.

6.5 Packets Forwarded

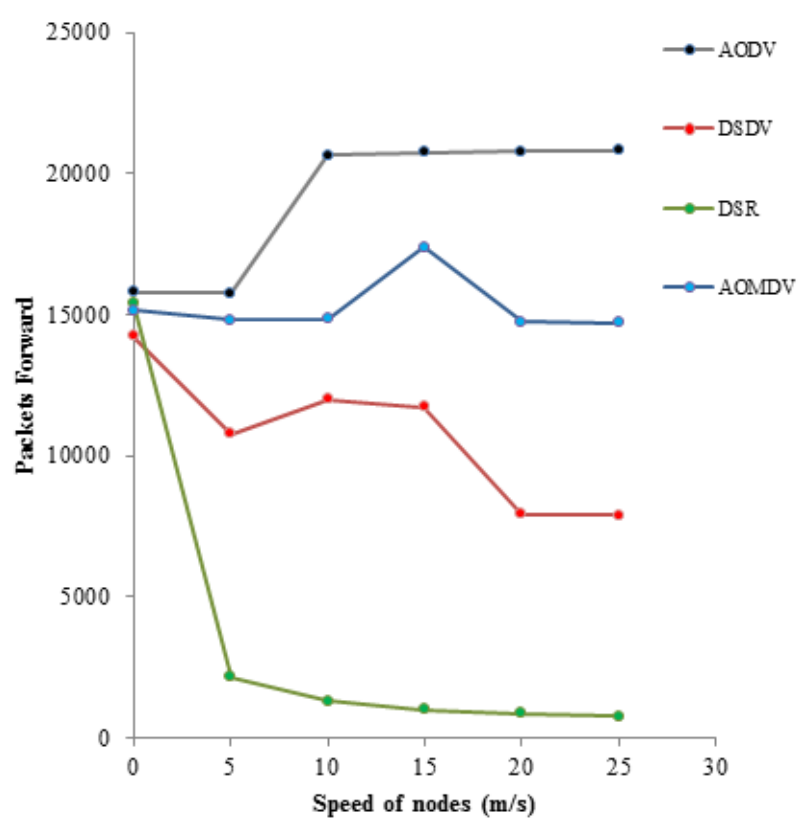

Fig 11: Packets sent vs. Speed of nodes for different routing protocol.

For forwarding packet AODV performs better as shown in Fig 11. For both DSDV and DSR the packet forward decreases with increasing the velocity of the mobile nodes. The amount of forwarded packet for AOMDV routing protocol is also good which is noticeable from the given figure.

The overall conclusion is that, for a MANET with mobile gateway, AOMDV is better choice with average performance.Throughput, Delay, Packet Delivery Ratio, Packets received and Packets sent parameters are used to analyze the protocols. It is not important that AOMDV performs always better for all the parameters, its performance may vary with different parameters which is observed in the case of Average End-to-End delay the performance of the 
DSR routing protocol is better than all other three protocols. At the end of simulation it is noticeable that the performance of the routing protocols varies with the performance parameters.

\section{IMPORTANCE OF THIS RESEARCH}

By using this Mobile Ad hoc Network a seamless heterogeneous communication can be established through mobile gateway which will be cost effective because there have no need to any base station and infrastructure. It would be a great feature for some emergency purposes such as to establish communication in a disaster area for rescuing purpose, to enhance the transmission range etc. The effectiveness in usage of bandwidth will appreciably increase. Opportunities for better information availability and sharing at anywhere and anytime will increase. By adopting the best routing protocol for this network the most reliable communication network can be built.

\section{CONCLUSIONS}

This paper attempts to define mobile ad hoc network architecture in a heterogeneous communication environment. Nowadays, the traffic in both aeronautical and nautical communication is increasing rapidly. So, new technological solutions have to be developed to meet the heavily loaded Internet traffic. Cost savings and operational improvements through better and greater access to aircraft data while on ground can be met by wireless broadband access to aircraft and flight data upon landing during turnarounds and layovers and use of low cost wireless technology to transfer large amounts of non-critical data. To meet the future requirements for robust heterogeneous communication network, interface among different types of communication network making is very important for which the aeronautical and nautical mobile Ad hoc network have to be adapted. Furthermore, future work will invovle the security issue of this network architecture. In this approach, it was already shown by considering various performance metrics that the performance of communication network dpends on the performance parameter. For establishing a good communication the AOMDV routing protocol is suggested by analyzing the performance for various performance parameters in our approach. Advanced research work on aeronautical and nautical Mobile Ad hoc Network with heavily loaded gateway by the Internet traffic can make a perfect robust communication network for both aeronautical and nautical environment.

\section{REFERENCES}

[1] D. Medina and F. Hoffmann, "The Airborne Internet" Future Aeronautical Communications, Edited by S. Plass, September 2011.

[2] P. Sharma, A. Kalia and J. Thakur, " Performance analysis of AODV, DSR AND DSDV routing protocols in mobile ad-hoc network (MANET)" Journal of Information Systems and Communication, Bioinfo Publications, Volume 3, Issue 1, 2012, pages: 322-326.

[3] I. Chlamtac, M. Conti, J. J.-N. Liu, "Mobile ad hoc networking: imperatives and challenges" Ad Hoc
Networks, Elsevier publication, Volume 1, Number 1, July 2003, pages: 13-64.

[4] F. Ulloa-Vásquez and J .A. Delgado-Penín, "Performance simulation in High Altitude Platforms (Haps) communications systems" IST Project Helinet and Spanish Government (MCYT) TIC2000-0320-CE, supported by the European Commission, available online at: http://www.haps.cl/documentos/13.pdf

[5] M. W. Soomro, M. A. Memon and M. I. Abro, "Performance analysis of routing protocols in MANET with static and mobile nodes using HTTP traffic" International Journal of Electronics and Communication Engineering (IJECE), Volume 1, Issue 2, Nov 2012, pages: $23-31$.

[6] W. Kiess, M. Mauve, "A survey on real-world implementations of mobile ad-hoc networks" Ad Hoc Networks, Elsevier publication, Volume 5, No. 3, April 2007, pages: 324-339.

[7] M. Hiyama, E. Kulla, T. Oda, M. Ikeda and L. Barolli, "Application of a MANET Testbed for horizontal and vertical scenarios: performance evaluation using delay and jitter metrics" Human-centric Computing and Information Sciences 2011, pages: 1-14.

[8] NEWSKY Project, European Commission Project, "Mobile aeronautical communication network based on internet technologies for cockpit and cabin services integrating satellite and terrestrial data links" German Aerospace Center, Feb. 2007-Oct. 2009, available online at: www.newsky-fp6.eu.

[9] S. R. Biradar, K. Majumder, S. K. Sarkar, Puttamadappa, "Performance evaluation and comparison of AODV and AOMDV" International Journal on Computer Science and Engineering (IJCSE), Volume 02, Issue 02, 2010, pages: $373-377$

[10] E. M. Royer and C. K. Toh, "A review of current routing protocols ad hoc mobile wireless networks" IEEE Personal Communications, Volume 6, Number 2, April 1999, pages: 46-55.

[11] Manual (formerly notes and documentation) of Network Simulator available online at http://www.isi.edu/nsnam/ns/index.html.

[12] TEL-WIMAX-NS2 project documentation, Computer and Electronics Engineering Department at the University of Nebraska-Lincoln , Peter Kiewit Institute in Omaha, Nebraska., (01.10.2012), available online at: http://www.TEL.unl.edu.

[13] "Simulation And Implementation", On-Demand Routing In Multi-Hop Wireless Mobile Ad Hoc Networks, India, (01.10.2012), pages: $73-138$ available online at http://shodhganga.inflibnet.ac.in/bitstream/10603/4106/1 3/13_chapter\%205.pdf 